\title{
Lipomatosis encefalocraneocutánea: reporte de caso
}

\author{
Juan Pablo Muñoz-Montufar', Jesús Darío Rayo-Mares', Araceli Reyes-Cuayahuitl'1, \\ Daniel Octavio Pacheco-Rosas y Mónica Paulina Rangel-Ramírez ${ }^{1}$ \\ ${ }^{1}$ Servicio de Neurología Pediátrica; ${ }^{2}$ Servicio de Infectología Pediátrica. Unidad Médica de Alta Especialidad Hospital de Pediatría Dr. Silvestre
} Freunk Freund, Centro Médico Nacional Siglo XXI, Instituto Mexicano del Seguro Social, Ciudad de México, México

\section{Resumen}

La lipomatosis encefalocraneocutánea (ECCL), también conocida como síndrome de Haberland o de Fishman, es un síndrome neurocutáneo raro y de etiología aún desconocida. Clínicamente se caracteriza por lesiones dérmicas, oculares y del sistema nervioso central. Se presenta el caso de una paciente de 7 años que acude a la consulta externa de neurología pediátrica por la presencia de crisis convulsivas, detectándose datos clínicos y de neuroimagen compatibles con ECCL. Nuestro objetivo es dar a conocer este caso por la rareza de la enfermedad y la variedad de alteraciones encontradas en la tomografía.

PALABRAS CLAVE: Lipomatosis encefalocraneocutánea. Síndrome de Haberland o de Fishman. Nevus psiloliparus.

\section{Abstract}

The encephalocraniocutaneous lipomatosis (ECCL), also known as Fishman or Haberland syndrome, is a rare neurocutaneous syndrome of unknown etiology. Clinically characterized by skin, eye and central nervous system lesions. We present the case of a 7-year-old female who presents to the outpatient clinic of Pediatric Neurology because of the presence of seizures detecting clinical and neuroimaging manifestations compatible with ECCL. The objective of this article is to present the case because of its rare presentation and the variety of alterations found in the tomography.

KEY WORDS: Lipomatosis encephalocraniocutaneous. Haberland or Fishman syndrome. Nevus psiloliparus.

\section{Introducción}

La lipomatosis encefalocraneocutánea (ECCL), también denominada síndrome de Haberland o síndrome de Fishman, es un síndrome cutáneo raro que característicamente involucra al tejido ectomesodérmico, como la piel, los ojos y el sistema nervioso central (SNC). La tríada clásica consiste en anormalidades oculares (nevus psiloliparus, lipomas subcutáneos en la región frontotemporal, hipoplasia o aplasia dérmica focal), anormalidades oculares (coristomas, colobomas, anormalidades corneales o de la cámara anterior, y calcificación del globo ocular) y anormalidades del SNC (lipomas intraespinales, asimetría de vasos intracraneales, atrofia hemisférica, dilatación ventricular, hidrocefalia, quistes aracnoideos, quistes porencefálicos o calcificaciones). La mayoría de los casos descritos son unilaterales; sin embargo, existen reportes de casos con afección bilateral'.

\section{Presentación del caso}

Presentamos el caso de una niña de 7 años sin antecedentes heredofamiliares, perinatales ni patológicos, con desarrollo psicomotor normal y con

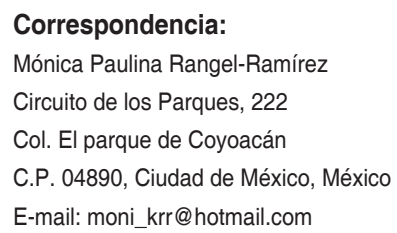

Fecha de recepción: 20-08-2016

Fecha de aceptación: 08-09-2016 DOI://dx.doi.org/10.24875/GMM.17002833
Gac Med Mex. 2017;153:915-918

Contents available at PubMed www.gacetamedicademexico.com 
adecuado desempeño escolar. Al nacimiento se detectó lesión en ojo derecho, así como endotropía, y en el canto externo del mismo ojo una lesión fibrosa. A los 6 meses de edad comienza con crisis convulsivas focales caracterizadas por desviación de la mirada hacia la derecha, por lo que inicia tratamiento con valproato de magnesio.

A la exploración presenta una zona de alopecia en la región frontal derecha, y en el canto externo una lesión fibrosa (Fig. 1). En la mejilla derecha se observa una lesión papular de aproximadamente $0.5 \mathrm{~mm}$, normocrómica (Fig. 2). En el ojo derecho, pupila con bradicoria, agudeza visual disminuida y limitación para la abducción por presencia de coristoma epibulbar (Fig. 3). El resto de la exploración, sin alteraciones aparentes.

Debido al antecedente de crisis convulsivas, se solicita electroencefalograma y se encuentra con asimetría por mayor amplitud y menor frecuencia en la región parietooccipital derecha (Fig. 4).

Se realizó tomografía de cráneo simple y contrastada, en la cual se detectó hemiatrofia cortico-subcortical, quiste aracnoideo temporal Galassi II que desplaza el ala mayor del esfenoides, que mide $5.8 \times 2.3 \mathrm{~mm}$, calcificaciones corticales parieto-occipitales, lipoma pontocerebeloso del lado derecho de $21 \times 10.6 \mathrm{~mm}$, con densidades de $-63 \mathrm{UH}$ y menor calibre de los vasos arteriales en el hemisferio cerebral derecho, y calcificación en coroides del globo ocular derecho; ninguna de las lesiones con realce tras la administración de contraste (Fig. 5).

\section{Discusión}

La ECCL (también conocida como síndrome de Haberland o de Fishman) es un síndrome neurocutáneo raro, encontrándose en la literatura unos 90 casos reportados ${ }^{2}$. Fue descrita por primera vez por Haberland y Perou en $1970^{3}$, y posteriormente Fishman contribuyó con la descripción de casos nuevos 4 .

Su etiología aún es desconocida y hasta el momento no se han detectado alteraciones cromosómicas; se ha considerado un mosaico probablemente de una mutación autosómica de un gen involucrado en la generación de tumores múltiples del mesénquima y de la vasculogénesis ${ }^{5}$. Se considera que la patogénesis es una disgenesia de la cresta neural cefálica y del tubo neural anterior, afectando predominantemente a los derivados del ectodermo y del mesodermo ${ }^{6}$.

Clínicamente se caracteriza por lesiones dérmicas, oculares y del SNC. La principal alteración dermatológica

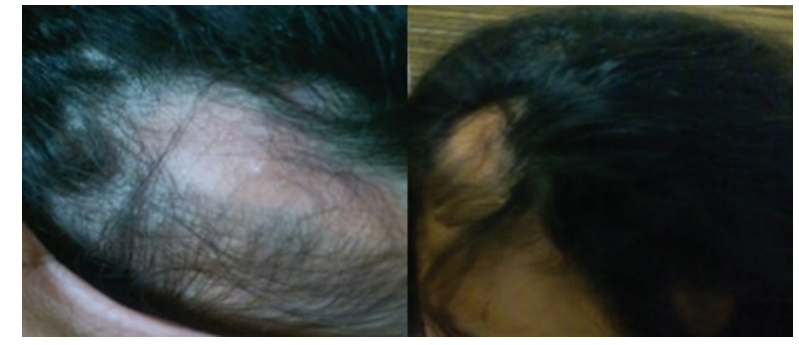

Figura 1. Alopecia no cicatricial en la región frontal derecha.

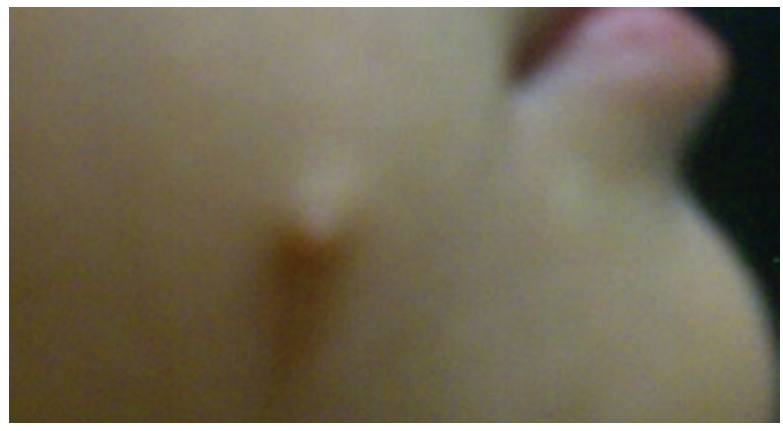

Figura 2. Lesión papular en la mejilla derecha.

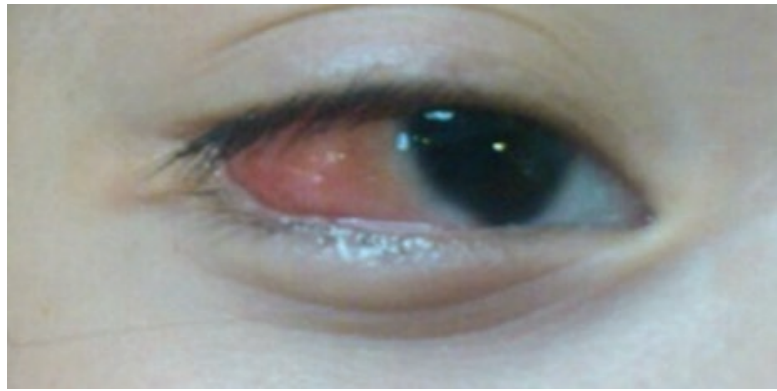

Figura 3. Coristoma epibulbar en el ojo derecho.

es el nevus psiloliparus ${ }^{7}$; también es típica la presencia en el mismo lado de la cara de lesiones papulonodulares normocrómicas de tamaño variable en la frente, la mejilla y el párpado, que pueden corresponder a lipomas, fibrolipomas, hamartomas o nevus de tejido conjuntivo ${ }^{8,9}$.

La principal alteración ocular son los coristomas ${ }^{1}$ homolaterales a las lesiones cutáneas, que pueden causar ambliopía al inducir opacidad corneal, defectos de refracción o ambos ${ }^{8,9}$. Puede haber otras anormalidades oculares, como colobomas, microftalmia, anormalidades de la cámara anterior y calcificaciones del globo ocular. Existen otras anormalidades más raras, como papiledema, epicanto inverso, displasia de iris e hipotelorismo ${ }^{10}$.

Las lesiones cerebrales se localizan en el mismo lado que las lesiones dérmicas, e incluyen hemiatrofia, lipomas intracraneales y medulares, quistes aracnoideos y porencefálicos, dilatación ventricular 


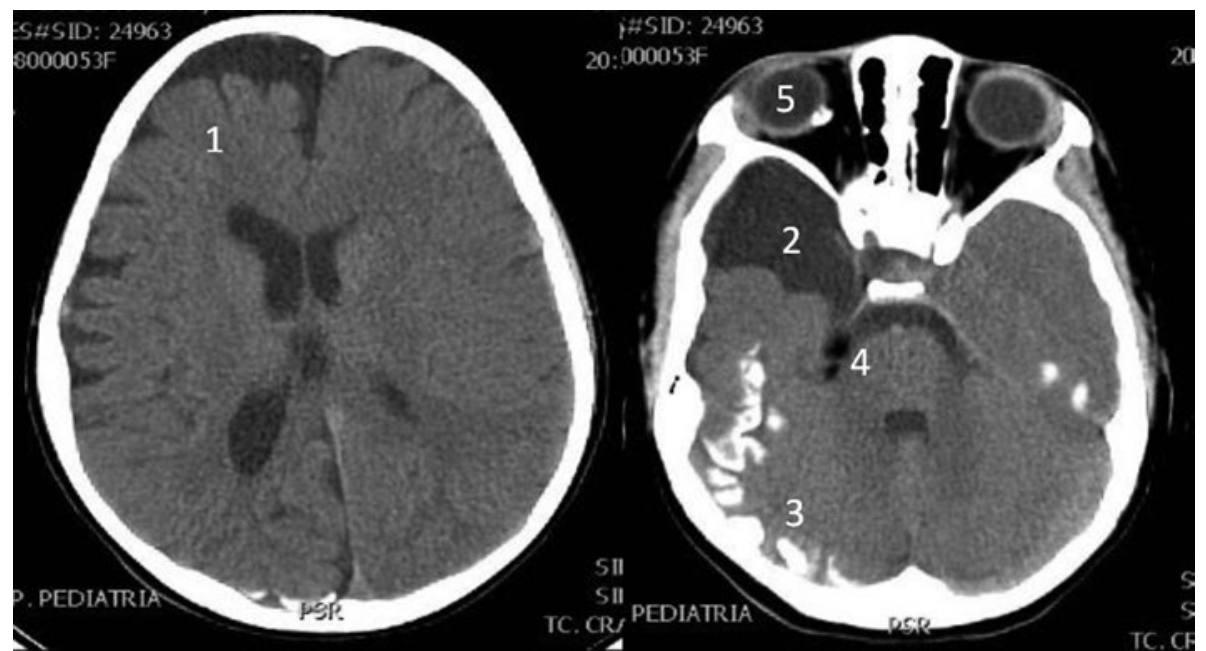

Figura 4. Electroencefalograma en el que se observa asimetría de amplitud interhemisférica y actividad delta occipital derecha.

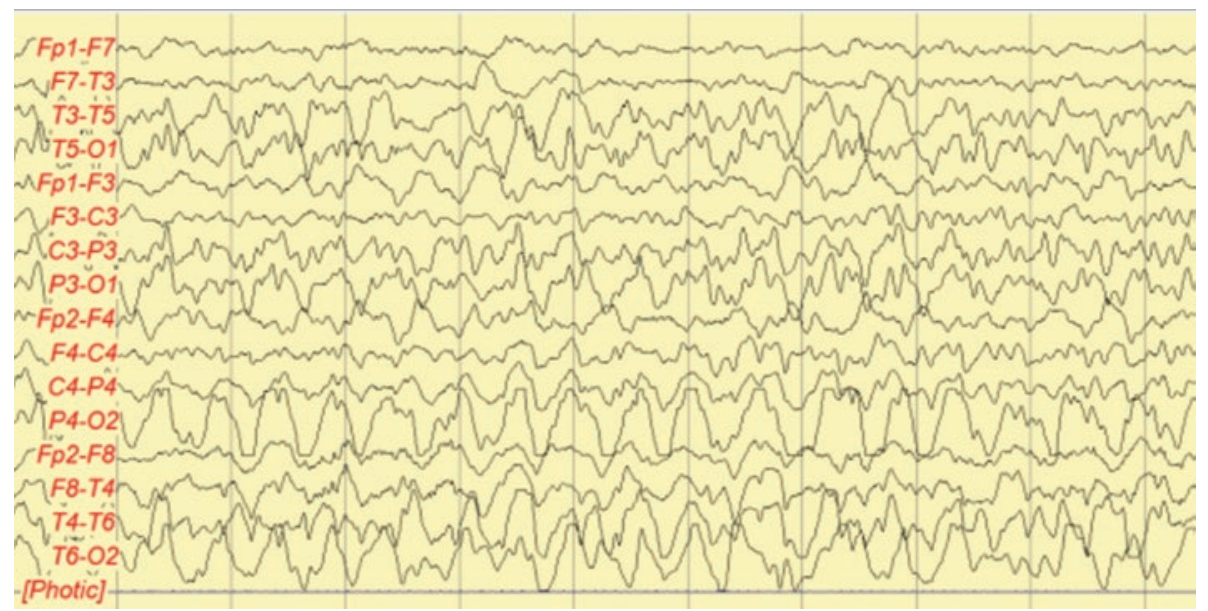

Figura 5. Tomografía de cráneo simple en cortes axiales en la que se observan hemiatrofia derecha (1), quiste aracnoideo temporal Galassi II (2), calcificaciones corticales parieto-occipitales (3), lipoma pontocerebeloso del lado derecho (4) y calcificación coroidea del ojo derecho (5).

adyacente e hidrocefalia. También puede haber pérdida de la normal opercularización de la ínsula y calcificaciones corticales, lipomas, adelgazamiento del cuerpo calloso y lesiones vasculares (anormalidades de los vasos y angiomatosis leptomeníngea) $)^{2,8}$. Pueden presentar discapacidad intelectual hasta un $40 \%$ y crisis convulsivas un $50-60 \%$ de los pacientes ${ }^{5,11}$, y otros como hemiplejia, parálisis facial, espasticidad, hipoacusia neurosensorial y trastornos de la conducta ${ }^{12}$.

El diagnóstico incluye la realización de biopsia de las lesiones de la piel, que demostrará grasa subcutánea en la dermis reticular. Están indicados estudios de neuroimagen y examen oftalmológico. Un ecocardiograma y un electrocardiograma descartarán problemas cardiacos. Originalmente, los criterios fueron propuestos por Hunter en $2006^{1}$, y posteriormente Moog ${ }^{5}$ realizó en 2009 una revisión de 54 casos con diagnóstico de ECCL y estableció los criterios clínicos para su diagnóstico (Tabla 1).

El diagnóstico diferencial de la enfermedad se establece con el síndrome oculoectodérmico, una condición caracterizada por coristomas, particularmente dermoides epibulbares y alopecia no cicatricial; sin embargo, en esta enfermedad la lesión en el cráneo es la aplasia cutis congénita, y los lipomas faciales e intracraneales son inusuales. El síndrome de Proteus es una condición multisistémica de aparición esporádica que puede presentarse también con lipomas cutáneos y coristomas oculares en una distribución en mosaico, aunque esta condición se caracteriza por un sobrecrecimiento desproporcionado y asimétrico sobre todo de los brazos, el cráneo y el meato auditivo externo. La lesión dérmica característica es un nevus cerebriforme de tejido conectivo, así como nevus epidérmicos múltiples, lipomas o lipohiplosia, y 
Tabla 1. Criterios diagnósticos de Moog para lipomatosis encefalocraneocutánea

\begin{tabular}{|c|c|c|c|}
\hline Alteraciones oculares & Alteraciones cutáneas & $\begin{array}{l}\text { Alteraciones en el sistema } \\
\text { nervioso central }\end{array}$ & Otras \\
\hline $\begin{array}{l}\text { Criterios mayores: } \\
\text { 1. Coristoma con o sin anomalías } \\
\text { asociadas } \\
\text { Criterios menores: } \\
\text { 1. Anormalidades corneales o de } \\
\text { cámara anterior } \\
\text { 2. Coloboma ocular o palpebral } \\
\text { 3. Calcificación ocular }\end{array}$ & $\begin{array}{l}\text { Criterios mayores: } \\
\text { 1. Nevus psiloliparus confirmado } \\
\text { 2. Posible nevus psiloliparus } \geq 1 \text { de los } \\
\text { criterios menores } 2-5 \\
\text { 3. } \geq 2 \text { criterios menores } 2-5 \\
\text { Criterios menores: } \\
\text { 1. Posible nevus psiloliparus } \\
\text { 2. Alopecia irregular no cicatricial (sin } \\
\text { nevus graso) } \\
\text { 3. Lipoma subcutáneo en la región } \\
\text { frontotemporal } \\
\text { 4. Aplasia o hipoplasia dérmica focal } \\
\text { sobre el escalpe } \\
\text { 5. Lesiones dérmicas nodulares en } \\
\text { párpados o sobre el canto externo o } \\
\text { el trago }\end{array}$ & $\begin{array}{l}\text { Criterios mayores: } \\
\text { 1. Lipoma intracraneal } \\
\text { 2. Lipoma intraespinal } \\
\text { 3. } \geq 2 \text { criterios menores } \\
\text { Criterios menores: } \\
\text { 1. Anormalidades vasculares } \\
\text { intracraneales, como angiomas } \\
\text { 2. Quiste aracnoideo u otras } \\
\text { anormalidades en las meninges } \\
\text { 3. Atrofia hemisférica completa } \\
\text { o parcial } \\
\text { 4. Quistes porencefálicos } \\
\text { 5. Dilatación ventricular } \\
\text { asimétrica o hidrocefalia } \\
\text { 6. Calcificaciones (no sobre } \\
\text { núcleos de la base) }\end{array}$ & $\begin{array}{l}\text { Criterios mayores: } \\
\text { 1. Tumor de } \\
\text { mandíbula (osteoma, } \\
\text { odontoma o fibroma osificante) } \\
\text { 2. Quistes óseos múltiples } \\
\text { 3. Coartación de aorta }\end{array}$ \\
\hline \multicolumn{4}{|c|}{$\begin{array}{l}\text { Caso definitivo: } \\
\text { 1. Involucro de tres sistemas, dos o más criterios mayores. } \\
\text { 2. Involucro de tres sistemas, nevus psiloliparus probado o posible con uno o más criterios menores en piel del } 2 \text { al } 5 \text {. } \\
\text { 3. Involucro de dos sistemas con criterio mayor; uno debe ser nevus psiloliparus probado, o si se trata de un posible nevus psiloliparus } \\
\text { debe incluir uno más de los criterios en piel del } 2 \text { al } 5 \text {. }\end{array}$} \\
\hline
\end{tabular}

malformaciones vasculares. El síndrome oculocerebrocutáneo o síndrome de Delleman se caracteriza por anormalidades congénitas oculares, cerebrales y cutáneas. Se distingue de la ECCL porque en el síndrome de Delleman existe un hamartoma de músculo estriado. Asimismo, se deberá realizar diagnóstico diferencial con otros síndromes, como los de Schimmelpenning, Goltz y Goldenhar ${ }^{1,9,13}$.

El tratamiento es sintomático, por lo que en caso de crisis convulsivas se pautarán fármacos antiepilépticos. Se puede recurrir al tratamiento estético quirúrgico para las lesiones dérmicas u oculares ${ }^{14}$.

\section{Conclusión}

El síndrome de Haberland es poco frecuente, existiendo alrededor de 90 casos reportados. Debe considerarse la ECCL ante la presencia de hallazgos clínicos dérmicos que incluyen alopecia no cicatricial, lesión papular en la mejilla y coristoma epibulbar; así como ante hallazgos radiológicos como los encontrados en nuestra paciente, incluyendo hemiatrofia, quistes aracnoideos, lipoma intracraneal, y calcificaciones corticales y ocular. Es importante considerar el diagnóstico a pesar de ser una enfermedad rara y probablemente subdiagnosticada.

\section{Bibliografía}

1. Hunter AG. Oculocerebrocutaneous and encephalocraniocutaneous lipomatosis syndromes: blind men and an elephant or separate syndromes? Am J Med Genet. 2006;140:709-26.

2. Moog U, Jones MC, Viskochil DH, et al. Brain anomalies in encephalocraniocutaneous lipomatosis. Am J Med Genet. 2007;143A:2963-72.

3. Haberland C, Perou M. Encephalocraniocutaneous lipomatosis: a new example of ectomesodermal dysgenesis. Arch Neurol. 1970;22:144-55.

4. Fishman MA, Chang CS, Miller JE. Encephalocraniocutaneous lipomatosis. Pediatrics. 1978;61:580-82.

5. Moog U. Encefphalocraniocutaneous lipomatosis. J Med Genet. 2009; 46:721-9.

6. Koishi GN, Yoshida M, Alonso N, et al. Encephalocraniocutaneous lipomatosis (Haberland's Syndrome) - A case report of a neurocutaneous syndrome and a review of the literature. Clinics. 2008;63:406-8.

7. Happle R, Horster S. Nevus psiloliparus: report of two nonsyndromic cases. Eur J Dermatol. 2004;14:314-6.

8. Brown KE, Goldstein SM, Douglas RS, et al. Encephalocraniocutaneous lipomatosis: a neurocutaneous syndrome. J AAPOS. 2003;7:148-9.

9. Rubegni $P$, Risulo M, Sbano $P$, et al. Encephalocraniocutaneous lipomatosis (Haberland syndrome) with bilateral cutaneous and visceral involvment. Clin Exp Dermatol. 2003;28:387-90.

10. Chittenden HB, Harman HE, Robinson F, et al. A case of encephalocraniocutaneous lipomatosis. Br J Ophtalmol. 2002;86:934-5.

11. Donaire A, Carreno $M$, Bargalló N, et al. Presurgical evaluation and cognitive functional reorganization in Fishman syndrome. Epilepsy Behav. 2005;6:440-3.

12. Chan CC, Chen JS, Chu CY. Haberland syndrome. Dermatol Sin. 2005; 23:41-5.

13. Cohen MM Jr. Proteus syndrome: an update. Am J Med C Semin Med Genet. 2005;137C:38-52

14. Chandravanshi S. Encephalocraniocutaneous lipomatosis: a case report and review of the literature. Indian J Ophthalmol. 2014;62:622. 